\title{
Seizures and epilepsy in the acute medical setting: presentation and management
}

\author{
Authors: Elizabeth Caruana Galizia ${ }^{\mathrm{A},}$ and Howard John Faulkner ${ }^{\mathrm{B},}$
}

Epileptic seizures are a common cause for presentation to acute medical services. Whether presenting with an isolated, unprovoked seizure or with status epilepticus, a good understanding of seizures and their mimics ensures appropriate investigation and treatment. This article describes the practical aspects of the management of patients presenting with seizures to the emergency department or the acute medical unit.

\section{Introduction}

Seizures are one of the commonest causes of transient loss of consciousness presenting to acute medical services and epileptic seizures are the commonest cause of short admissions among the neurological conditions. While not all seizures are associated with lost consciousness, most requiring acute medical care will involve impaired awareness and responsiveness. Approximately $1 \%$ of the UK population are treated for epilepsy, with around 0.5 new cases per 1,000 population every year. ${ }^{1}$ Acute medical and emergency department physicians need a good working knowledge of the management of seizures and to be aware of seizure mimics.

\section{Transient loss of consciousness}

Transient loss of consciousness (T-LOC) is a common complaint that may present to many different specialties under various labels, including 'blackouts', 'seizure', 'blank spells', and 'falls'. The term T-LOC itself is non-specific and includes all causes of selflimited loss of consciousness, regardless of mechanism. ${ }^{3}$ Syncope is the commonest cause of T-LOC, followed by epileptic seizures and non-epileptic seizures. ${ }^{3}$

A thorough history including a witness account will allow a diagnosis to be made in the majority of patients. ${ }^{4}$ Distinguishing seizures from syncope and functional non-epileptic seizures can be difficult, but these are equally important diagnoses to make. Patients with functional non-epileptic seizures are often exposed to inappropriate interventions and treatment with antiepileptic drugs. Diagnosing functional non-epileptic attack disorder and its management are covered in detail elsewhere in this issue. Syncope

Authors: A consultant neurologist, Department of Neurology, Atkinson Morley Wing, St George's Hospital, London, UK; ' ${ }^{\text {C }}$ consultant neurologist, Department of Neurology, Southmead Hospital, Bristol, UK; *authors contributed equally may be associated with a high mortality rate, likely reflecting a high-risk subgroup of patients ${ }^{5}$ and not uncommonly presents with myoclonic jerks, head turning, and other motor phenomena often associated with epileptic seizures; ${ }^{6}$ Sheldon et al, ${ }^{7}$ proposed a series of questions that they demonstrated would distinguish seizures from syncope with $94 \%$ sensitivity and specificity (see Table 1).

Raised prolactin (within $30 \mathrm{~min}$ ) or lactate following loss of consciousness can contribute to the distinction between bilateral tonic-clonic seizures and syncope or a non-epileptic attack, but are less helpful in the differentiation of non-convulsive episodes and are generally not recommended. ${ }^{8,9}$ Where the history is lacking, atypical, or inconclusive, early involvement of specialists experienced in the investigation and management of T-LOC improves diagnosis. ${ }^{10}$

\section{First seizures}

According to the National Institute for Health and Care Excellence (NICE) guidance, ${ }^{11}$ anyone with a suspected first seizure should be referred for specialist assessment and seen within 2 weeks. Most patients with a single selfterminating seizure who have made a full recovery can be managed as outpatients through local first-seizure pathways.

\section{Key points}

Transient loss of consciousness is common, with syncope, epileptic seizures, and non-epileptic seizures accounting for most presentations

History, including a witness account where possible, is key

Consider urgent brain imaging in specific circumstances

Seizures may be provoked or unprovoked and identifying the underlying cause has implications for treatment and onward referral

Status epilepticus is a medical emergency; timely treatment, delivered in appropriate doses are paramount

KEYWORDS: seizures, epilepsy, status epilepticus, transient loss of consciousness, classification 
Table 1. The Sheldon questionnaire. The patient has seizures if point score $\geq 1$, and syncope if score is $<1$. The same questions can be asked of a witness

Questions shown to distinguish seizures from syncope

At times do you wake with a cut tongue after your spells? 2

At times do you have a sense of déjà vu or jamais vu 1 before your spells?

At times is emotional stress associated with losing 1 consciousness?

Has anyone noted your head turning during a spell? 1

Has anyone ever noted that you are unresponsive, have unusual posturing or have jerking limbs during your spells or have no memory of your spells afterwards? (Score as yes for any positive response)

Has anyone ever noted that you are confused after a spell? 1

Have you ever had lightheaded spells? -2

At times do you sweat before your spells? $\quad-2$

Is prolonged sitting or standing associated with your spells? - -2

Reproduced with permission?

Antiepileptic medication is not indicated for a single seizure, unless investigations indicate a high risk of seizure recurrence. ${ }^{12}$ Other than in status epilepticus, starting antiepileptic drugs should ideally be a specialist decision. In the interim, the patient should be given basic safety and driving advice.

> Driving: by law patients must refrain from driving until specialist assessment. If a seizure is confirmed, the Driver and Vehicle Licensing Agency (DVLA) must be informed and a group 1 licence will usually be revoked for a minimum of 6 to 12 months (depending on the presence/absence of abnormalities on magnetic resonance imaging [MRI] / electroencephalogram [EEG]).

> Safety and lifestyle: shower rather than bath; avoid heights / dangerous equipment; occupational/parenting guidance; first aid in the event of recurrent events; and who to contact.

\section{Investigations: who should have urgent brain imaging and who should be admitted?}

All patients with a first seizure presentation should be investigated with routine blood tests to exclude infection or metabolic disturbance and should have an electrocardiogram (ECG). The majority of uncomplicated first seizure patients do not require urgent brain imaging before discharge. MRI is the imaging modality of choice to investigate seizures and will be organised via a first seizure clinic. Urgent brain imaging (preferably with MRI, but with computed tomography [CT] if MRI is not available / feasible acutely) before discharge should be considered in those patients where an acute intracranial event is suspected (see Box 1).

Patients with abnormal imaging, prolonged or recurrent events, or incomplete recovery may justify a brief admission for more urgent (inpatient) medical and neurological assessment. ${ }^{13}$
Box 1. Indications for urgent brain imaging and/or

hospital admission

Indications for urgent brain imaging ${ }^{a}$ and/or hospital admission

Acute head trauma

New onset focal neurologic deficit

Altered mental status persists (behaviour or cognition)

Recurrent events

Persistent headache

Anticoagulation

A history of immunodeficiency or malignancy

Fever

Focal seizure (partial seizure)

New neurological symptoms prior to the seizure

Patients in whom follow-up cannot be ensured

a Urgent imaging is not required for syncope, non-epileptic seizures, or patients with well-characterised epilepsy or recurrent admissions with drug/alcoholprovoked seizures in the absence of any additional reason to suspect new intracranial pathology

\section{Acute symptomatic seizures}

This encompasses all seizures that occur in close association with a brain insult. The International League Against Epilepsy (ILAE) has proposed specific parameters where seizures can be called acute symptomatic (see Table 2). ${ }^{14}$ In these circumstances treatment should not be initiated unless there are multiple seizures, or if the patient is in status epilepticus, followed by prompt specialist input with respect to continuation. The risk of seizure recurrence is often minimal so long-term treatment with antiepileptic drugs is not required.

\section{Seizures in known epilepsy}

This is an extremely common scenario. Patients can present reporting deterioration in their usual frequency for a range of reasons including:

> patient/family anxiety about perceived changes

$>$ intercurrent infections / systemic illnesses

$>$ alcohol and recreational drug use

> medication related: eg poor adherence, changes in formulation/ brand, during planned withdrawal / changes to medication,

drug interactions with other prescribed and over the counter medications

> new acute symptomatic seizures, for example due to a head injury.

The history is key. Is the seizure pattern unusual for the patient? Ask how often seizures occur at their worst or their best. Confusion about the patient's normal seizure pattern can occur in new environments, for example, a stranger may have called an ambulance unnecessarily. If the above precipitants have been excluded, fluctuations in seizure frequency may occur simply due to the natural variability of epilepsy.

If adherence has been poor, try to establish why, in order to address the root cause (eg side effects needing a change in drug/ dose, forgetfulness - would a blister pack help?) 
Table 2. Parameters for acute symptomatic seizures proposed by the International League against Epilepsy

\begin{tabular}{|c|c|}
\hline Provoking insult & Time-frame \\
\hline Stroke/hypoxia & $<1$ week \\
\hline $\begin{array}{l}\text { Traumatic brain injury without } \\
\text { subdural haematoma }\end{array}$ & $<1$ week \\
\hline $\begin{array}{l}\text { Traumatic brain injury with subdural } \\
\text { haematoma }\end{array}$ & Up to 1 month \\
\hline Intracranial surgery & $<1$ week \\
\hline $\begin{array}{l}\text { Arteriovenous malformation at time } \\
\text { of haemorrhage }\end{array}$ & $<1$ week \\
\hline CNS infection & $\begin{array}{l}\text { Until laboratory and } \\
\text { clinical signs of infection } \\
\text { have resolved }\end{array}$ \\
\hline Multiple sclerosis & <1 week (of relapse) \\
\hline Alcohol withdrawal & $\begin{array}{l}7-48 \text { hours from last } \\
\text { alcoholic drink }\end{array}$ \\
\hline Serum glucose (within 24 hours) & $\begin{array}{l}<36 \mathrm{mg} / \mathrm{dL}(2 \mathrm{mM}) \text { or } \\
>450 \mathrm{mg} / \mathrm{dL}(25 \mathrm{mM}) \text { and } \\
\text { ketoacidosis }\end{array}$ \\
\hline Serum sodium (within 24 hours) & $<115 \mathrm{mg} / \mathrm{dL}(<5 \mathrm{mM})$ \\
\hline Serum calcium (within 24 hours) & $<5 \mathrm{mg} / \mathrm{dL}(<1.2 \mathrm{mM})$ \\
\hline Serum magnesium (within 24 hours) & $<0.8 \mathrm{mg} / \mathrm{dL}(<0.3 \mathrm{mM})$ \\
\hline Urea nitrogen (within 24 hours) & $>100$ mg/dL (>35.7 mM) \\
\hline Creatinine (within 24 hours) & $>10$ mg/dL (>884 uM) \\
\hline
\end{tabular}

Adapted with permission from Beghi et al, $2010 .{ }^{14} \mathrm{CNS}=$ central nervous system

Investigations: ECG and screening bloods for sepsis and metabolic causes should include antiepileptic drug (AED) levels where possible to assess adherence. Try to resist the temptation for brain imaging unless there is a clear clinical indication (see Box 1) and EEG is rarely useful in this situation.

In addition to addressing the cause, management may involve simple reassurance.

Seek advice from the patient's usual treating team or local neurology services, but try to avoid escalating the dose of existing medications without specialist advice. If short-term rescue medications are required for safe discharge, consider using clobazam $10 \mathrm{mg}$ at night as a temporary add-on therapy prior to specialist advice.

\section{Status epilepticus}

Status epilepticus (SE) is defined by 2 time points: the first time point is when a seizure has failed to self-terminate and has become 'abnormally prolonged' leading to the second time point when there is a risk of neurological damage and longterm consequences. SE may be convulsive or non-convulsive. ${ }^{15}$ Convulsive SE (CSE) is a medical emergency, operationally defined by tonic clonic seizures persisting (or recurring without recovery) beyond $5 \mathrm{~min}$, with an increasing risk of morbidity and mortality if not controlled by $30 \mathrm{~min}$. Treatment should be initiated at $5 \mathrm{~min}$.

Most hospitals will have local protocols for the treatment of convulsive status epilepticus. A typical protocol is shown in Fig 1: all involve initial treatment with benzodiazepines (in adults typically up to 2 doses of $4 \mathrm{mg}$ of lorazepam or $10 \mathrm{mg}$ midazolam, which may include pre-hospital treatment by carers or paramedics, ${ }^{11}$ followed by AEDs for ongoing seizures. Phenytoin and phenobarbitone are the only AEDs currently licensed in this situation, with speed and adequate dosing $(20 \mathrm{mg} / \mathrm{kg}$ for phenytoin, $15 \mathrm{mg} / \mathrm{kg}$ for phenobarbitone) probably being more important than choice of agent.

Meta-analyses suggest equipotency of valproate $(30-40 \mathrm{mg} /$ $\mathrm{kg}$, $\max 3 \mathrm{~g}$ ) and levetiracetam $(40-60 \mathrm{mg} / \mathrm{kg}$, max $4.5 \mathrm{~g}$ ) with phenytoin, although these are not yet licensed for use in SE. ${ }^{17}$ Presently there is insufficient evidence to recommend either drug ahead of existing therapies for management of SE pending the outcome of ongoing trials. ${ }^{18}$ Those with ongoing seizures despite appropriate treatment need urgent intervention with anaesthetic agents and management on an intensive care unit with local neurology input.

Non-convulsive status epilepticus (NCSE) is a more difficult diagnosis usually made based on EEG. Unlike CSE where early intervention is essential, aetiology is the principal determinant of outcome in NCSE rather than duration of seizures and thus escalating treatment prior to EEG is not indicated without specialist advice. ${ }^{16}$ Treatment usually involves the use of intravenous benzodiazepines under EEG control.

\section{Classification}

The classification of seizures and the epilepsies has recently been updated. A comprehensive review of the new classification is available on the ILAE website (www.ilae.org). Increasingly, one will see, and should start to use, the following terms in clinical practice: ${ }^{20}$

> 'partial' is replaced by the term 'focal'; this can be further qualified by whether awareness is retained or not, so that 'complex partial' becomes 'focal unaware' and 'simple partial' becomes 'focal aware'

> 'secondarily generalised' is replaced by 'focal to bilateral tonic clonic'

$>$ for generalised onset seizures, seizures can be described as 'generalised motor', which would include tonic clonic seizures, or 'generalised non-motor', which refers to 'absences'

> 'unknown onset' and 'unclassified' have been added, acknowledging that, not uncommonly, seizure onset and aetiology cannot be defined.

\section{Conclusion}

Seizures and seizure-mimics are common presentations to emergency departments and acute medical units, either as isolated events or as a medical emergency in status epileptics. The history is key in the former situation, while speed in delivering appropriate treatment cannot be over-emphasised in the latter. Central to this is early input from the neurology or epilepsy team, that will guide appropriate investigation and, if indicated, the start of treatment. 
Immediate measures

Fig 1: Algorithm for the treatment of convulsive status epilepticus. Adapted with permission from the American Epilepsy Society guideline $2016 .{ }^{19}$ The most important differential diagnosis of status epilepticus is nonepileptic seizures. Non-epileptic seizures tend to last longer than epileptic seizures and should be considered as a diagnosis before emergency antiepileptic drugs are administered. Alongside treatment, urgent investigations must be performed to confirm the cause of the status epilepticus. CA2+ = calcium; $\mathrm{FBC}=$ full blood count; IV = intravenous; $\mathrm{LFT}=$ liver function test; $\mathrm{Mg} 2+=$ magnesium; $\mathrm{U}+\mathrm{E}=$ urea and electrolytes; $\mathrm{IM}=$ intramuscular

20-40 $\mathrm{min}$

\section{Stabilise patient}

Secure airway (recovery position) and give oxygen Call for help

Monitor vital signs and institute cardiac monitoring

Establish IV access and take venous blood samples for glucose, LFT, U+E, Mg2+, CA2+, FBC, toxicology screening and antiepileptic drug levels

If there are concerns about hypoglycaemia, poor nutrition or alcohol excess, give $\mathbf{2 5 0}$ $\mathrm{mg}$ of thiamine IV, followed by $50 \mathrm{~mL}$ of $\mathbf{5 0 \%}$ glucose IV over 10 minutes (consider $1 \mathrm{mg}$ IM glucagon if IV access not available)

\section{Give IV lorazepam $0.1 \mathrm{mg} / \mathrm{kg}$ (max $4 \mathrm{mg}$ ) over a few seconds}

If lorazepam is not available give diazepam $10 \mathrm{mg}$ IV $(0.15 \mathrm{mg} / \mathrm{kg}$ )

If IV access is not possible give buccal midazolam $10 \mathrm{mg}$

If no response repeat after $\mathbf{1 0} \mathrm{min}$ and inform intensive care unit (ITU)

\section{If no response}

\section{Give phenytoin IV $(20 \mathrm{mg} / \mathrm{kg})$}

or one of:

\section{phenobarbital IV $(10-15 \mathrm{mg} / \mathrm{kg})$}

sodium valproate IV (30-40 $\mathrm{mg} / \mathrm{kg}$, max dose $3,000 \mathrm{mg}$ )

levetiracetam IV (40-60 mg/kg, max dose 4,500 $\mathrm{mg}$ )

If no response

Refractory status epilepticus

Intubate, give general anaesthesia and admit to ITU

\section{References}

1 Joint Epilepsy Council. Prevalence, incidence and other statistics. JEC, 2011. www.epilepsyscotland.org.uk/pdf/Joint_Epilepsy_Council_ Prevalence_and_Incidence_September_11_\% 283\% 29.pdf

2 van Dijk N, Boer KR, Colman N et al. High diagnostic yield and accuracy of history, physical examination, and ECG in patients with transient loss of consciousness in FAST: the Fainting Assessment study. J Cardiovasc Electrophysiol 2008;19:48-55

3 Task Force for the Diagnosis and Management of Syncope, European Society of Cardiology (ESC), European Heart Rhythm Association (EHRA), Heart Failure Association (HFA) et al. Guidelines for the diagnosis and management of syncope (version 2009). Eur Heart ] 2009;30:2631-71.

4 Reuber M, Chen M, Jamnadas-Khoda J et al. Value of patientreported symptoms in the diagnosis of transient loss of consciousness. Neurology 2016;87:625-33.

5 Soteriades ES, Evans JC, Larson MG et al. Incidence and prognosis of syncope. N Engl J Med 2002;347:878-85.

6 Lempert T, Bauer M, Schmidt D. Syncope: a videometric analysis of 56 episodes of transient cerebral hypoxia. Ann Neurol 1994:36:233-7.

7 Sheldon R, Rose S, Ritchie D et al. Historical criteria that distinguish syncope from seizures. J Am Coll Cardiol 2002;40:142-8.
8 Matz O, Zdebik C, Zechbauer S et al. Lactate as a diagnostic marker in transient loss of consciousness. Seizure 2016;40:71-5.

9 Chen DK, So YT, Fisher RS, Therapeutics and Technology Assessment Subcommittee of the American Academy of Neurology. Use of serum prolactin in diagnosing epileptic seizures: report of the Therapeutics and Technology Assessment Subcommittee of the American Academy of Neurology. Neurology 2005;65:668-75.

10 Briggs R, Coughlan T, Doherty ] et al. Investigation and diagnostic formulation in patients admitted with transient loss of consciousness. Ir Med J 2017:110:563.

11 National Institute for Health and Clinical Excellence. Epilepsies: diagnosis and management [CG137]. London: NICE, 2012.

12 Marson A, Jacoby A, Johnson A et al. Immediate versus deferred antiepileptic drug treatment for early epilepsy and single seizures: a randomised controlled trial. Lancet Lond Engl 2005;365:200713.

13 Dunn MJG, Breen DP, Davenport RJ, Gray AJ. Early management of adults with an uncomplicated first generalised seizure. Emerg Med ] 2005:22:237-42.

14 Beghi E, Carpio A, Forsgren L et al. Recommendation for a definition of acute symptomatic seizure. Epilepsia 2010;51:671-5.

15 Trinka E, Cock H, Hesdorffer D et al. A definition and classification of status epilepticus - Report of the ILAE task force on classification of status epilepticus. Epilepsia 2015;56:1515-23. 
16 Ferguson M, Bianchi MT, Sutter R et al. Calculating the risk benefit equation for aggressive treatment of non-convulsive status epilepticus. Neurocrit Care 2013;18:216-27.

17 Yasiry Z, Shorvon SD. The relative effectiveness of five antiepileptic drugs in treatment of benzodiazepine-resistant convulsive status epilepticus: a meta-analysis of published studies. Seizure 2014:23:167-74

18 Bleck T, Cock H, Chamberlain J et al. The Established Status Epilepticus Trial 2013. Epilepsia 2013;54:89-92.

19 Glauser T, Shinnar S, Gloss D et al. Evidence-based guideline: treatment of convulsive status epilepticus in children and adults: report of the guideline committee of the American Epilepsy Society. Epilepsy Curr 2016;16:48-61.
20 Fisher RS, Cross JH, French JA et al. Operational classification of seizure types by the International League Against Epilepsy: position paper of the ILAE Commission for Classification and Terminology. Epilepsia 2017:58:522-30.

Address for correspondence: Dr Howard John Faulkner, Department of Neurology, Brunel Building, Southmead Hospital, Bristol BS10 5NB, UK.

Email: Howard.Faulkner@nbt.nhs.uk

\section{4ing:? Royal College Q. of Physicians}

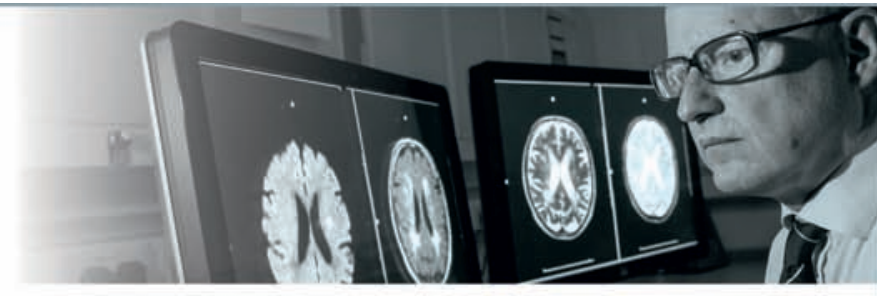
Medical Care

\section{Are you involved in planning neurology services?}

Medical Care is the online evolution of the well-known RCP publication Consultant physicians working with patients. Written by leading medical specialty experts, it is a comprehensive web-based resource for the efficient and effective design of medical services covering all the specialties, including neurology.

\section{www.rcpmedicalcare.org.uk}

The resource looks in detail at the services delivered by neurology, including:

$>$ regional neurosciences centres

$>$ acute services

$>$ stroke services

$>$ outpatient care

$>$ transition services.

Medical Care has been designed to help those involved in the planning and provision of medical services to get a clearer picture of the specialty services that need to be in place to provide great patient care. 